\title{
Post-operative small pelvic field radiation therapy in patients with intermediate risk early stage cervix cancer: a safe and efficient treatment modality
}

\author{
José Antonio Solis ${ }^{1,2}$, Ilan Perrot Rosenberg ${ }^{(1,2}{ }^{1,}$ Jorge Olivares ${ }^{1,2}$, Benjamin Tudela ${ }^{1,2}$, Gabriel Veillon ${ }^{1,2}$, \\ Gabriel Lazcano ${ }^{1,2}$ \\ 'Radiation Oncology Department, Hospital Carlos Van Buren, City of Valparaíso, Valparaíso Region, Chile \\ ${ }^{2}$ Universidad de Valparaíso, Faculty of Medicine, Reñaca, Viña del Mar, Valparaíso Region, Chile
}

\begin{abstract}
Background: The treatment of early stage cervical cancer has different therapeutic options. Adjuvant external beam radiotherapy for surgically treated intermediate risk cervical cancer patients has shown acceptable oncological outcomes with a low incidence of toxicity. The aim of this study was to analyze the oncological outcomes and safety of adjuvant small pelvic field radiotherapy in surgically treated stage IB1-2 cervical cancer patients who met the Sedlis intermediate-risk criteria.

Materials and methods: A retrospective cohort study was carried out with 28 patients treated from 2007 to November 2019 with biopsy proven intermediate risk stage IB1-2 cervical cancer previously treated with radical hysterectomy and bilateral lymphadenectomy who received adjuvant small pelvic field radiotherapy. The primary end points were local and distant control and overall survival. Secondary endpoints were acute and late gastrointestinal and genitourinary toxicity. Survival curves were analyzed using the Kaplan-Meier method.

Results: After a median follow up period of 41.5 (27.5-80.5) months, adjuvant small pelvic field radiotherapy showed a $100 \%$ overall survival rate, $81.82 \%$ disease free survival and $86.36 \%$ local recurrence-free survival with no incidence of grade 3 or 4 acute or late toxicity. Three patients suffered from relapse, 1 in the vaginal cuff, 1 in the retrovesical area and 1 patient in the retroperitoneal area.

Conclusions: Adjuvant small pelvic field radiotherapy is an efficient and safe treatment option that offers excellent oncological outcomes to surgically treated intermediate-risk stage IB1-2 cervical cancer patients with an excellent toxicity profile.

Key words: cervical cancer; small pelvic field; intermediate risk; 3D conformal radiotherapy

Rep Pract Oncol Radiother 2021;26(3):360-366
\end{abstract}

\section{Introduction}

Cervical cancer is the most common gynecologic cancer worldwide [1], more common in low- and middle-income countries. It is the second most common cancer in incidence among women and the third most common cancer in terms of mortal- ity [2]. The treatment of early stage cervical cancer (FIGO stage IB2) has different options described in the literature, both primary radiotherapy or surgery followed by radiotherapy with or without chemotherapy have been validated in different settings [2].

High-risk disease includes patients with either positive surgical margins, lymph node metastases

Address for correspondence: Ilan Perrot Rosenberg MD, Servicio de Oncología Hospital Carlos Van Buren, Hontaneda 2560, Valparaíso, Chile, tel: +569 91823013; e-mail: iperrotr@gmail.com

This article is available in open access under Creative Common Attribution-Non-Commercial-No Derivatives 4.0 International (CC BY-NC-ND 4.0) license, allowing to download articles and share them with others as long as they credit the authors and the publisher, but without permission to change them in any way or use them commercially 
or parametrial spread, and such patients should be offered postoperative radiotherapy with chemotherapy since the intergroup study 0107 (GOG 109/SWOG 8797/RTOG 91-12) demonstrated an advantage in overall survival when patients receive this adjuvant treatment [3]. Surgical pathological studies performed by the Gynecologic Oncology Group [4-7], identified risk factors that were categorized as intermediate (tumor size greater than 4 $\mathrm{cm}$, lymph vascular invasion and stromal invasion deep). In the absence of the previously described high risk factors, this group of patients benefit from adjuvant radiotherapy alone $[6,7]$

The classic whole-pelvic field radiotherapy (WPRT) is commonly used in most postoperative radiotherapy treatments. In this setting, the absence of the uterus causes the bowel loops to fall into the pelvis, increasing toxicity. It seems reasonable to state that directing radiotherapy exclusively to the small pelvis would be less toxic than WPRT, without compromising oncological outcomes since most recurrences occur within the small pelvis [8]. This pattern is also supported by the observed nodal spread in some surgical series, where the obturator, internal and external iliac (83.7\%) are the most common sentinel nodes in early cervical cancer [9], followed by the common iliac lymph nodes (6.6\%), paraaortic (2\%) and inguinal (0.07) \% [10]. So, the most common nodal involvement sites are covered within our small pelvic field.

Reports have shown that small field pelvic radiotherapy offers satisfactory results [11-13] showing, as well, a considerable reduction in treatment-related morbidity [11-16]. In the present study, postoperative cervical cancer patients who met the Sedlis [6] criteria and received adjuvant small field pelvic radiotherapy were retrospectively analyzed to investigate its clinical efficacy and safety.

\section{Materials and methods}

\section{General clinical data}

Patients treated for cervical cancer between January 2007 and November 2019 at the Carlos Van Buren Hospital, Valparaiso, Chile, were eligible for this study. The study inclusion criteria are: $\geq 18$ years of age, diagnosed with early stage cervical cancer (FIGO $2009 \leq$ IB2), presenting at least 2 intermediate risk factors (tumor size greater than $4 \mathrm{~cm}$, lymph vascular invasion and deep stro- mal invasion), managed with radical hysterectomy plus bilateral lymphadenectomy followed by small pelvic field radiotherapy at our institution. We excluded patients with high-risk features, as well as those who had not been treated or followed by a gynecologist oncologist.

The primary end points were to determine the local and distant control defined as percentage of patients without evidence of relapse within the pelvis and distant organs as well as overall survival. This definition encompasses relapses happening from the first day of radiotherapy treatment to the date of the last medical appointment. Secondary outcomes included toxicity, both acute and late genitourinary and gastrointestinal, according to the RTOG $[17,18]$ toxicity grading system. Acute toxicity was considered when adverse events occurred within 90 days following the start of radiotherapy and late toxicity later than 90 days.

\section{Radiotherapy protocol}

Patients were treated with 3D conformal radiotherapy using a small pelvic 4-field box technique, without brachytherapy. Clinical target volume (CTV) was defined as the vaginal cuff with a 2 $\mathrm{cm}$ margin, also including regional lymphnodes (bilateral internal iliac, external iliac and obturator chains) up to the inferior border of the sacroiliac joint. Planning target volume (PTV) was created with an isotropic expansion of $1 \mathrm{~cm}$. A visual example of the treatment set up can be found in Figure 1. All patients were treated with 6-18 MV photon beam in a Varian 21iX Linear accelerator with an 80 multileaf collimator. Dose prescription ranged from 45 to 50.4 Gray in $1.8 \mathrm{G}$ to 2 Gy daily fractions. Treatments were planned using Eclipse 8.6.23. Follow up was every 4 months during the first year, every 6 months up to 5 years and then annually.

\section{Study design}

For this single retrospective cohort, information was extracted from the clinical records of early stage cervical cancer (FIGO $\leq$ IB2) patients who met the inclusion criteria and received radiotherapy with a small pelvic field at the radiation oncology unit in the Carlos Van Buren hospital. Our research was previously approved by the local ethics committee. The data were obtained by reviewing the records available in the clinical files of our facility. 


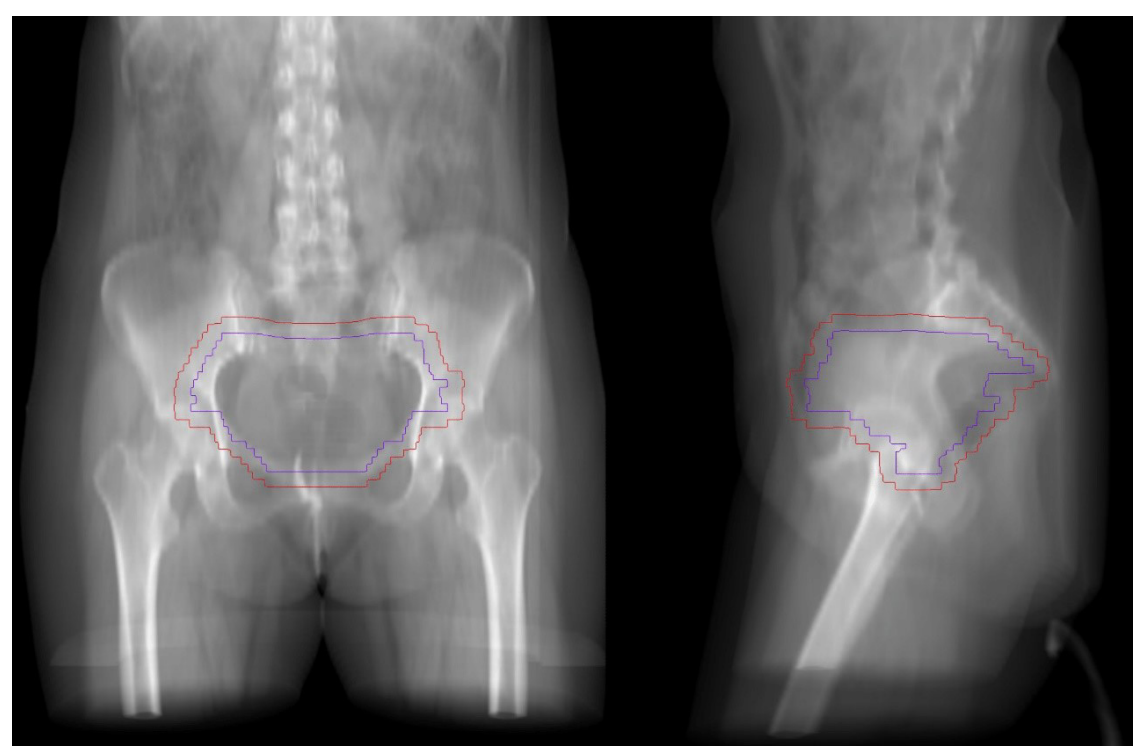

Figure 1. Treatment set up. Purple — clinical target volume (CTV); red — planning target volume (PTV)

\section{Statistical analysis}

All statistical analysis was performed using STATA 16.1 SE (StataCorp LLC, College Station, TX, United States of America). Survival analysis was made with the Kaplan-Meier method, estimating overall survival, disease-free survival, and local recurrence-free survival, all defined from the start of radiation therapy. Patients were censored at last follow-up visit if they had no evidence of recurrent disease. Treatment toxicities were graded under the RTOG toxicity grading system $[17,18]$.

\section{Results}

Between January 2007 and November 2019, 1529 patients were treated for cervical cancer at our center. Inclusion criteria were met by 29 patients; however, 1 patient was excluded because of incomplete surgery, not executed by a gynecological oncologist (no lymphadenectomy). The most prescribed radiotherapy dose was $50.4 \mathrm{~Gy}$ in 28 fractions to the PTV, although 2 patients received 45 Gy and 48.6 Gy, respectively. The main characteristics of the studied patients are described in Table 1.

Median follow up time was 41.5 months (IQR 27.5-80.5). More than $70 \%$ of our population was under 50 years of age. The most common histological type was squamous cell carcinoma (78.6\%). The median number of lymph nodes resected was 21 (14.5-27). Median interval time between the surgery and radiotherapy was 48.5 days (IC 48.5-88.5),
27 patients were treated with radical hysterectomy (96.4\%). Median tumor size was $2.8 \mathrm{~cm}$ (IC 2.5-3.2) with only 2 patients presenting tumors larger than $4 \mathrm{~cm}$ (7.15\%). Stromal invasion was classified as superficial in 2 patients (7.14\%); intermediate in 11 patients (39.3\%) and deep in 10 patients (17.9\%). Lymph vascular invasion was present in 23 patients (82.1\%).

Regarding acute toxicity, 15 patients had no GI toxicity (53.5\%), 6 patients (21.4\%) suffered from grade 1 GI toxicity and only 7 patients $(25 \%)$ from grade 2. No patients had grade 3 or 4 GI toxicity in our study. Genitourinary toxicity was observed as follows: 18 patients with no toxicity (64.2\%), 9 patients with grade $1(32.14 \%)$ and only 1 patient with grade 2 toxicity (3.5\%). Regarding late toxicity, 26 patients $(96.29 \%)$ had no late GI toxicity and only one patient $(3.7 \%)$ showed grade I toxicity with mild digestive symptoms. Genitourinary toxicity incidence was also low, with 25 asymptomatic patients (93\%), 1 patient (3.7\%) with grade I toxicity and 1 patient $(3.7 \%)$ with grade 2 toxicity (mild occasional bleeding). Acute and late toxicities can be seen in Figures 2 and 3, respectively.

Three patients presented recurrent disease. Two of these events where local recurrences. One patient relapsed within the vaginal cuff 2 years after treatment; 1 patient in the retro vesical area 4 years after treatment; and 1 patient suffered from a retroperitoneal relapse located left from the L3-L4 junction. Five-year overall survival was $100 \%$ and 
Table 1. Patients characteristics

\begin{tabular}{|c|c|}
\hline Characteristic & Patients, n (\%)* \\
\hline Age, years & Median 47, IQR 36.5-56 \\
\hline$\leq 50$ & $17(60.7)$ \\
\hline$>50$ & $11(39.3)$ \\
\hline \multicolumn{2}{|l|}{ Type of surgery } \\
\hline Radical hysterectomy & $27(96.4)$ \\
\hline Trachelectomy & 1 (3.6) \\
\hline Resected lymph nodes & Median 21, IQR 14.5-27 \\
\hline $\begin{array}{l}\text { Interval between surgery } \\
\text { and radiotherapy, days }\end{array}$ & Median 48.5, IQR 48.5-88.5 \\
\hline Prescribed radiation dose, Gray & Median 50.4, IQR 50.4-50.4 \\
\hline Radiotherapy length, days & Median 39, IQR 37.5-41.5 \\
\hline Tumor size, centimeters & Mean 2.86, Cl 2.5-3.2 \\
\hline$\leq 2$ & $5(17.9)$ \\
\hline $2-4$ & $21(75)$ \\
\hline$>4$ & $2(7.15)$ \\
\hline \multicolumn{2}{|l|}{ Histopathology } \\
\hline Squamous cell carcinoma & $22(78.6)$ \\
\hline Adenocarcinoma & $3(10.7)$ \\
\hline Adenosquamous carcinoma & $2(7.1)$ \\
\hline Clear cell carcinoma & $1(3.6)$ \\
\hline \multicolumn{2}{|l|}{ Histologic grade } \\
\hline I & $6(21.4)$ \\
\hline II & $11(39.3)$ \\
\hline III & $5(17.9)$ \\
\hline N/A & $6(21.4)$ \\
\hline \multicolumn{2}{|l|}{ Cervical stromal invasion } \\
\hline Up to $1 / 3$ & $2(7.14)$ \\
\hline Up to $2 / 3$ & $11(39.3)$ \\
\hline Up to $3 / 3$ & $10(35.7)$ \\
\hline N/A & $5(17.9)$ \\
\hline $\begin{array}{l}\text { Lymph vascular space } \\
\text { involvement }\end{array}$ & $23(82.1)$ \\
\hline
\end{tabular}

${ }^{*}$ Or central tendency measures where appropriate; IQR — interquartile range; $\mathrm{Cl}$ - confidence interval

5-year disease-free survival was $81.82 \%$ (CI 95\%: 51.9-94.03), with a local recurrence-free survival of 86.36\% (CI 95\%: 53.61-96.61) (Fig. 4).

\section{Discussion}

Small pelvic field radiotherapy is a valid technique with excellent oncological outcomes and comparable with other conventional treatments in surgically managed cervical cancer patients with intermediate risk factors.

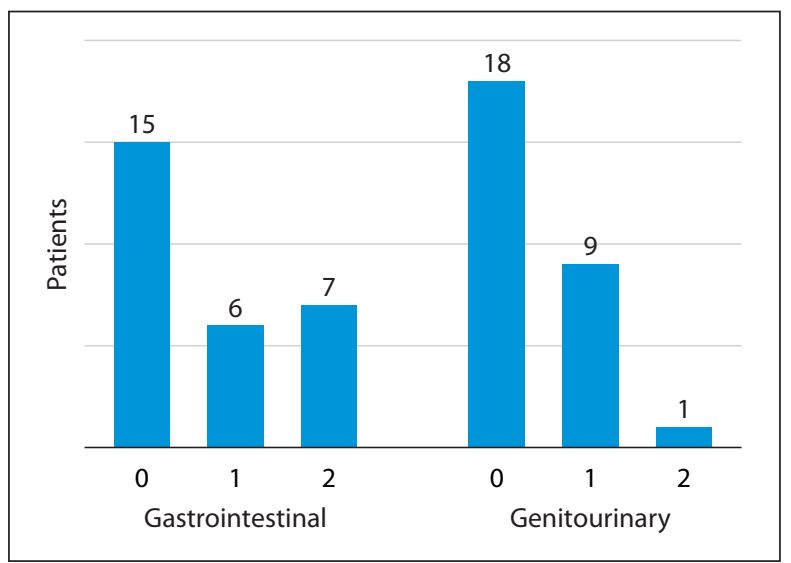

Figure 2. Bar graph reporting radiotherapy acute toxicity in the treated population for both gastrointestinal and genitourinary complications using the RTOG grading system

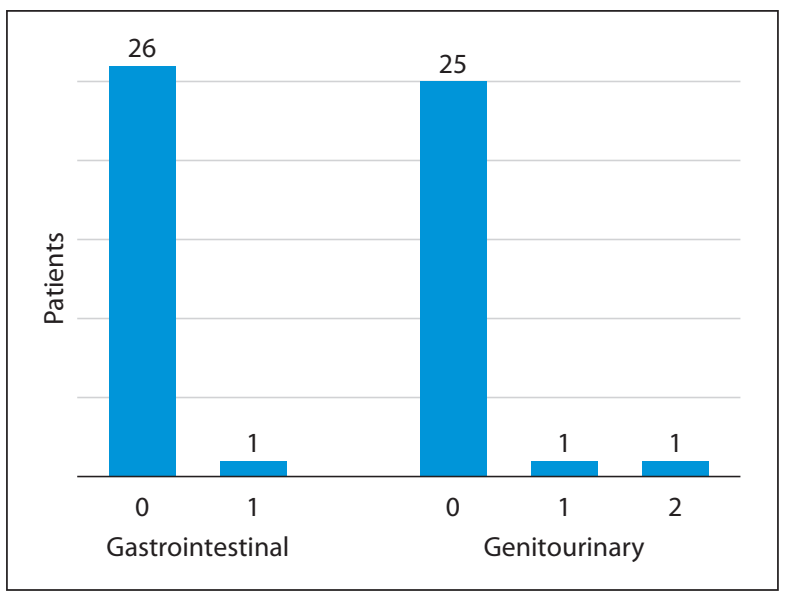

Figure 3. Bar graph reporting radiotherapy late toxicity in the treated population for both gastrointestinal and genitourinary complications using the RTOG grading system

Relevant publications and their outcomes are summarized in Table 2. We found that our results are comparable with those of Kridelka et al. [12] who used small pelvic field in 25 intermediate risk patients with only 1 local recurrence. In this study, the upper limit of the field was located in the S1-S2 intersection. In addition, the study demonstrated a significant improvement in 5-year DFS for the group that received adjuvant small field pelvic radiation compared to the observation group. Hong et al. [13] investigated whether postoperative radiation therapy to the lower pelvis is an appropriate treatment for patients with early high-risk cervical cancer with negative nodes. Seventy-nine patients 


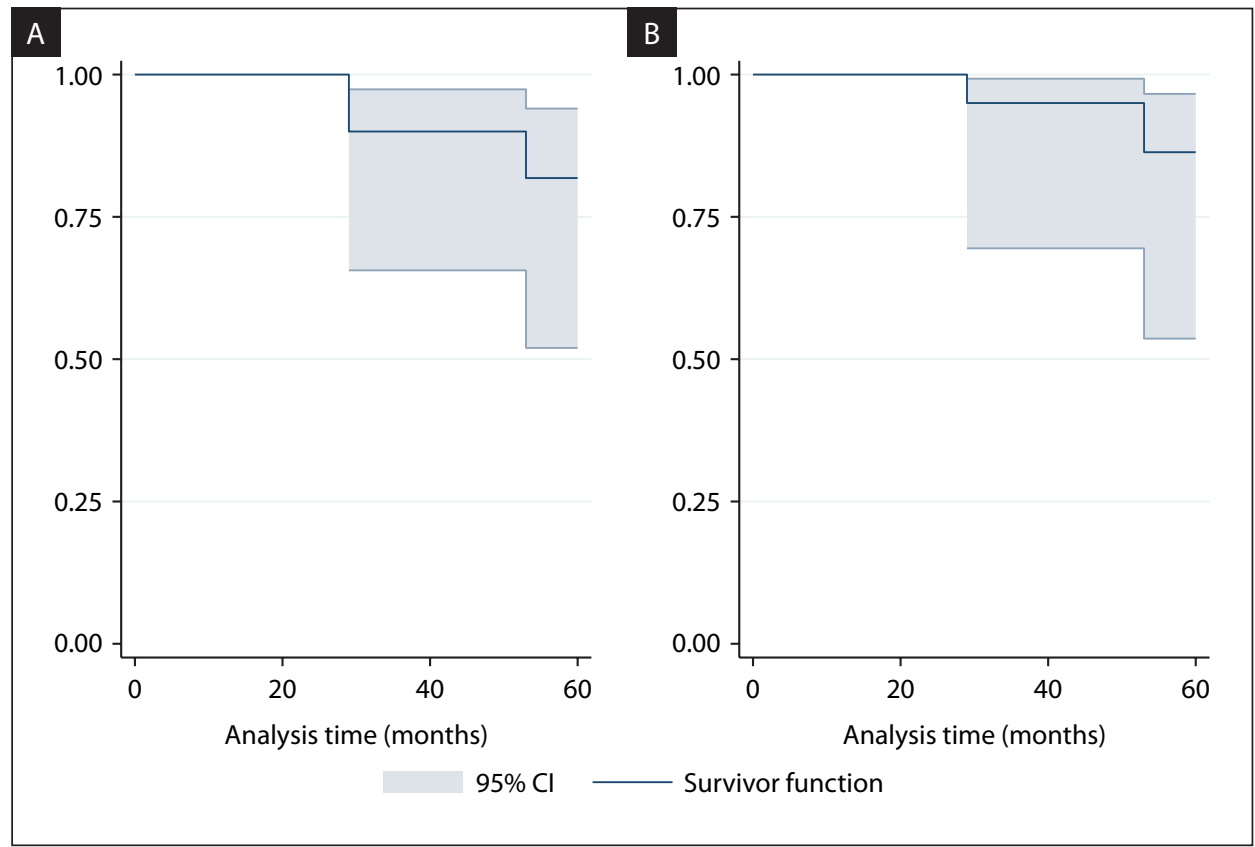

Figure 4. A. Five-year disease-free; B. Local recurrence-free survival

Table 2. Summary of small pelvic field radiation studies

\begin{tabular}{|c|c|c|c|c|c|}
\hline Treatment & Author & Stage & No. of patients & End point 1 & End point 2 \\
\hline Adjuvant SPRT alone & Kridelka et al. & IB negative nodes & 25 & DFS at $5 \mathrm{y}: 96 \%$ & \\
\hline $\begin{array}{l}\text { Adjuvant RT low pelvis } \\
\text { vs. Whole pelvis }\end{array}$ & Hong et al. & I to II negative nodes & 228 & OS at 5 y: $84 \%$ & DSS at 5 y: $86 \% *$ \\
\hline Adjuvant SPRT vs. WPRT & Ohara et al. & T1b-T2a & 72 & $\begin{array}{c}\text { 5-year pelvic disease } \\
\text { control rate for node- } \\
\text {-negative patients: } 93 \% *\end{array}$ & \\
\hline IMRT SPRT vs. WPRT & Luo et al. & $\mathrm{IA} 1-\mathrm{IB} 1$ & 371 & OS at $5 y: 91.6 \%$ & DFS at $5 \mathrm{y}: 89.3 \% *$ \\
\hline $\begin{array}{l}\text { Adjuvant SPRT } \\
\text { alone }\end{array}$ & Sari et al. & $\mathrm{IA} 2-\| \mathrm{A} 2$ & 113 & OS at 5 y: $82 \%$ & DFS at 5 y: $74 \%$. \\
\hline \multicolumn{6}{|c|}{ Adjuvant SPRT alone Present study lb — Intermediate risk 28} \\
\hline
\end{tabular}

SPRT — small pelvic field radiation therapy; WPRT — whole pelvis radiation therapy; DFS — disease-free survival; OS — overall survival; DSS — disease-specific survival. *No significant difference between the treatment arms

received whole-pelvic radiotherapy and a boost dose in the lower pelvis and 149 patients received low-pelvic RT alone. Results showed a 5-year overall and disease-specific survival rate of $84 \%$ and $86 \%$, respectively. In comparison, our results revealed more favorable outcomes, which can be explained by the fact that we only treated intermediate risk patients. Ohara et al. [11] examined whether the use of a small pelvic field reduces the adverse events that occur with the classic whole pelvic field. The 5 -year pelvic disease control rate for node-negative patients and node-positive patients was $93 \%$ versus 90\%, respectively. Acute diarrhea (Grades 2-3) occurred significantly more often in the whole pelvic group (32.4\%) compared to the small pelvic field group (9.2\%). Regarding late events, only ileus occurred at a significantly higher rate in the whole pelvic group than in the small pelvic field group (16.2\% vs. $3.2 \%)$. In our cohort no episodes of ileus were reported. Although both studies were retrospective, their results show similar local pelvic control with reduced treatment-related toxicity. Sari et al. [16] investigated whether small field adjuvant radiation therapy is sufficient in patients with early cervical cancer with intermediate risk factors. They retrospectively evaluated 113 patients, and all were treated with small-field pelvic RT. The upper limit of the field was the L5-S1 intervertebral space, but 
the lower edge is just inferior to the iliac foramina; that is, relatively smaller than conventional fields. The results showed an excellent pelvic control rate of $90.3 \%$ at 2 years with no severe gastrointestinal toxicity. These results are comparable to ours regarding disease control and safety. This finding supports that small field adjuvant pelvic RT is an adequate and sufficient treatment for patients with intermediate risk factors, in addition to providing clinical safety. At the same time, we highlight the fact that we used even tighter limits regarding field coverage without compromising pelvic control, as seen in our results.

In order to identify optimal candidates for small pelvic field radiotherapy Yeo et al. [14] performed a retrospective analysis of cervical cancer patients with FIGO stage IB to IIA who had undergone radical hysterectomy and lymphatic dissection. Patients without high risk factors would be classified according to the GOG score. Based on the score obtained, only observation, small-field radiation therapy, or standard field radiation therapy was recommended as obtaining excellent outcomes. Luo et al. [15] evaluated 371 patients with early cervical cancer, with at least one of the Sedlis criteria and without high risk factors for recurrence. All patients were treated with intensity modulated radiotherapy. Two hundred thirty-nine patients underwent whole pelvic radiotherapy, and 132 had reduced-volume pelvic radiotherapy. Incidence of upper and lower gastrointestinal symptoms with whole-pelvis radiotherapy was 94.5 and $96.6 \%$, respectively, compared with 71.2 and $81.8 \%$ for reduced-volume pelvic radiotherapy. Morbidity of this treatment appears to be acceptably low and significantly less than that associated with standard field pelvic radiation. This proves that even with the use of more sophisticated technologies, such as IMRT, the treatment volume in the pelvis remains an important prognostic factor for gastrointestinal toxicity. Currently, there are no studies comparing small field radiotherapy using 3DCRT vs IMRT, although it would be hard to justify the use of intensity modulated therapy with such a low toxicity incidence using 3D treatment.

Our work sets up a starting point to validate a simple technique suitable for other treatment modalities in the future, such as hypofractionation. In this scenario, patients could be treated in shorter periods of time, and if the treated volumes are redu- ced, the odds of both acute and late toxicity should be reduced.

Only one of the recurrences occurred outside the CTV, with a location left from the L3-L4 vertebral junction. It should be noted that this location would also be missed by a conventional, whole-pelvis treatment plan, with an upper limit for the CTV at the L4-L5 junction.

The weakness of our work is based on the retrospective nature of its design, and a limited number of included patients since the studied scenario is an uncommon one. As a strength, all of our patients were treated and followed by gynecologist-oncologists and we only considered their follow up appointments as valid when they were carried out at cervical pathology units so that all the obtained data from follow up visits was registered by expert physicians.

\section{Conclusion}

We conclude that external beam small pelvic field radiotherapy without brachytherapy offers excellent oncological outcomes showing comparable local and distant control to the other conventional treatments in surgically managed cervical cancer patients with intermediate risk factors. Since the execution of the technique and the equipment needed are highly available, we support small pelvic field radiotherapy (RT) as an alternative modality to reduce toxicity in centers which do not count with more modern RT techniques such as IMRT.

\section{Conflicts of interest}

The authors whose names are listed on the first page certify that they have NO affiliations with or involvement in any organization or entity with any financial interest (such as honoraria; educational grants; participation in speakers' bureaus; membership, employment, consultancies, stock ownership, or other equity interest; and expert testimony or patent-licensing arrangements), or non-financial interest (such as personal or professional relationships, affiliations, knowledge or beliefs) in the subject matter or materials discussed in this manuscript.

\section{Funding}

None declared. 


\section{Authors contribution}

All the authors of this manuscript collaborated in conceiving and designing the analysis. I.P.R. J.O, J.A.S. and B.T. collected the data and wrote the paper. The analysis was performed by J.A.S., I.P.R. and G.L., G.V. contributed in previous data and part of the analysis. All the authors listed above approve the final version of this article.

\section{References}

1. Bray F, Ferlay J, Soerjomataram I, et al. Global cancer statistics 2018: GLOBOCAN estimates of incidence and mortality worldwide for 36 cancers in 185 countries. CA Cancer J Clin. 2018; 68(6): 394-424, doi: 10.3322/ caac.21492, indexed in Pubmed: 30207593.

2. Bhtla N, Denny L. FIGO Cancer Report 2018: Cancer of the cervix uteri. Int J Gynaecol Obstet. 2018; 143(Suppl. 2): 22-36, doi: 10.1002/ijgo.12608, indexed in Pubmed: 30306587.

3. Peters WA, Liu PY, Barrett RJ, et al. Concurrent chemotherapy and pelvic radiation therapy compared with pelvic radiation therapy alone as adjuvant therapy after radical surgery in high-risk early-stage cancer of the cervix. J Clin Oncol. 2000; 18(8): 1606-1613, doi: 10.1200/ JCO.2000.18.8.1606, indexed in Pubmed: 10764420.

4. Delgado G, Bundy BN, Fowler WC, et al. A prospective surgical pathological study of stage I squamous carcinoma of the cervix: A Gynecologic Oncology Group study. Gynecol Oncol. 1989; 35(3): 314-320, doi: 10.1016/00908258(89)90070-x, indexed in Pubmed: 2599466.

5. Delgado G, Bundy B, Zaino R, et al. Prospective surgicalpathological study of disease-free interval in patients with stage IB squamous cell carcinoma of the cervix: A Gynecologic Oncology Group study. Gynecol Oncol. 1990; 38(3): 352-357, doi: 10.1016/0090-8258(90)90072-s, indexed in Pubmed: 2227547.

6. Sedlis A, Bundy BN, Rotman MZ, et al. A randomized trial of pelvic radiation therapy versus no further therapy in selected patients with stage IB carcinoma of the cervix after radical hysterectomy and pelvic lymphadenectomy: A Gynecologic Oncology Group Study. Gynecol Oncol. 1999; 73(2): 177-183, doi: 10.1006/gyno.1999.5387, indexed in Pubmed: 10329031.

7. Rotman M, Sedlis A, Piedmonte MR, et al. A phase III randomized trial of postoperative pelvic irradiation in Stage IB cervical carcinoma with poor prognostic features: follow-up of a gynecologic oncology group study. Int J Radiat Oncol Biol Phys. 2006; 65(1): 169-176, doi: 10.1016/j. ijrobp.2005.10.019, indexed in Pubmed: 16427212.
8. Look KY, Rocereto TF. Relapse patterns in FIGO stage IB carcinoma of the cervix. Gynecol Oncol. 1990; 38(1): 114-120, doi: 10.1016/0090-8258(90)90021-c, indexed in Pubmed: 2354816.

9. Balaya V, Guani B, Magaud L, et al. Long-term oncological safety of sentinel lymph node biopsy in early-stage cervical cancer. J Clin Oncol. 2020; 38(15 (Suppl.)): 6006-6006, doi: 10.1200/jco.2020.38.15_suppl.6006.

10. Ouldamer L, Marret $H$, Acker $O$, et al. Unusual localizations of sentinel lymph nodes in early stage cervical cancer: a review. Surg Oncol. 2012; 21(3): e153-e157, doi: 10.1016/j. suronc.2012.04.003, indexed in Pubmed: 22608843.

11. Ohara K, Tsunoda H, Satoh T, et al. Use of the small pelvic field instead of the classic whole pelvic field in postoperative radiotherapy for cervical cancer: reduction of adverse events. Int J Radiat Oncol Biol Phys. 2004; 60(1): 258-264, doi: 10.1016/j.ijrobp.2004.02.023, indexed in Pubmed: 15337564.

12. Kridelka FJ, Berg DO, Neuman M, et al. Adjuvant small field pelvic radiation for patients with high risk, stage IB lymph node negative cervix carcinoma after radical hysterectomy and pelvic lymph node dissection. A pilot study. Cancer. 1999; 86(10): 2059-2065, indexed in Pubmed: 10570432.

13. Hong JH, Tsai CS, Lai CH, et al. Postoperative low-pelvic irradiation for stage I-IIA cervical cancer patients with risk factors other than pelvic lymph node metastasis. Int J Radiat Oncol Biol Phys. 2002; 53(5): 1284-1290, doi: 10.1016/ s0360-3016(02)02831-6, indexed in Pubmed: 12128131.

14. Yeo RMC, Chia YN, Namuduri RPD, et al. Tailoring adjuvant radiotherapy for stage IB-IIA node negative cervical carcinoma after radical hysterectomy and pelvic lymph node dissection using the GOG score. Gynecol Oncol. 2011; 123(2): 225-229, doi: 10.1016/j.ygyno.2011.06.040, indexed in Pubmed: 21782226.

15. Luo HC, Lin GS, Liao SG, et al. Cervical cancer treated with reduced-volume intensity-modulated radiation therapy base on Sedlis criteria (NCCN VS RTOG). Br J Radiol. 2018; 91(1081): 20170398, doi: 10.1259/bjr.20170398, indexed in Pubmed: 29072851.

16. Yuce Sari S, Guler OC, Gultekin M, et al. Adjuvant Small Pelvic Radiotherapy in Patients with Cervical Cancer Having Intermediate Risk Factors Only - Is It Sufficient? Oncol Res Treat. 2017; 40(9): 523-527, doi: 10.1159/000476037, indexed in Pubmed: 28848218.

17. Perez CA, Brady LW. Acute Radiation Morbidity Scoring Criteria (RTOG). In: Perez CA, Brady LW. ed. Principles and practice of radiation oncology. 2nd ed. Lippincott, Philadelphia 1993: 51-53.

18. Perez CA, Brady LW. Late Radiation Morbidity Scoring Criteria (RTOG, EORTC). In: Perez CA, Brady LW. ed. Principles and practice of radiation oncology. 2nd ed. Lippincott, Philadelphia 1993: 53-55. 